\title{
CNS tuberculoma mimicking brain metastasis
}

\author{
Vinod K.S. Gautam ${ }^{1 *}$, Ankur Shrivastava ${ }^{2}$, Akriti Khare ${ }^{3}$, Ravinder Singh ${ }^{4}$ \\ ${ }^{I}$ Assistant Professor, Department of Neurosurgery, Institute of Human Behavior \& Allied Sciences, Dilshad Garden, \\ Delhi, India-110095 \\ ${ }^{2}$ Senior Resident, Department of Neurosurgery, Institute of Human Behavior \& Allied Sciences, Dilshad Garden, Delhi, India-110095 \\ ${ }^{3}$ Senior Resident, Department of Pathology, Delhi State Cancer Institute, Dilshad Garden, Delhi, India-110095 \\ ${ }^{4}$ Associate Professor \& Head, Department of Medical Anthropology, Institute of Human Behavior \& Allied Sciences, \\ Dilshad Garden, Delhi, India-110095 \\ *Corresponding author E-mail: drvksg@gmail.com
}

\begin{abstract}
A 32 year old male patient, a follow up case of carcinoma tongue post radiotherapy, presented with history of non-healing ulcer on lateral aspect of neck since 2 years and recurrent episodes of seizures for about 13 months duration. Neuroimaging was suggestive of metastasis in brain and patient was referred to us for biopsy of the lesion. Detailed clinical and radiological work up followed by neurosurgical intervention established the diagnosis of neurotuberculosis. In a patient with primary systemic malignanacy with suspected central nervous system metastasis, brain tuberculoma should always be kept as a differential diagnosis.
\end{abstract}

Keywords: Brain Metastasis, Carcinoma Tongue, Tuberculoma.

\section{Introduction}

Tuberculomas are the common intracranial space occupying lesions in countries where Tuberculosis (TB) is endemic (Gautam, Singh, Khurana, 2013; Gautam, Khurana, Singh, 2013; Ramamurthy, 1970). Brain metastases occur in up to $30 \%$ of patients with systemic malignancies (Norden,Wen,Kesari, 2005). Modern neuroradiological investigations help in early detection and treatment of brain metastases. But, the presentation of central nervous system (CNS) tuberculoma may also resemble with brain metastasis. This becomes more confusing in cancer patients or in cases which have been treated for primary malignancies. In such cases, the neurosurgical intervention for biopsy of the lesion is the procedure of choice to establish the diagnosis of CNS tuberculosis or metastasis.

\section{Case report}

A 32 year old male was a diagnosed as a case of carcinoma tongue and was treated with radiotherapy about two and half years back. He developed cervical lymphadenopathy on right side of neck while undergoing radiotherapy for carcinoma tongue. Later, his neck swelling ulcerated and this non-healing painless ulcer with underlying lymphadenopathy was persisting since 2 years. About, one year ago patient also had generalized tonic clonic type sei zures (GTCS) and seizures were recurrent inspite of taking antiepileptic drug (AED) for one year. Fine needle aspiration cytology (FNAC) of cervical lymph nodes was not conclusive of carcinoma. Positron emission tomography (PET) Scan [Fig.1] was suggestive of metastasis in cervical lymphnodes. So, patient was diagnosed as a case of carcinoma tongue with metastasis and put on chemotherapy. After one year, while on chemotherapy for metastasis, patient developed seizures. CT scan [Fig.2] and MRI brain [Fig.3] was reported as left frontal tuberculoma but in view of strong possibility of metastasis chemotherapy was continued. However, patient continued to have seizures despite of AEDs and then patient was eventually referred to our institute for management of brain lesion. A detailed clinical and radiological workup was done to reach a conclusive diagnosis. To control seizures another antiepileptic, Clobazam was added along with Phenytoin. Patient's chest X-Ray did not reveal any evidence of metastasis or pulmonary tuberculosis. Erythrocyte sedimentation rate (ESR) level was raised. MR Spectroscopy (MRS) was done which was suggestive of tuberculoma [Fig.4]. In view of a positive PET-CT and strong possibility of metastasis a neurosurgical intervention was planned. Patient underwent left frontal craniotomy and resection of intracranial lesion. Histopathology was suggestive of tuberculoma and antitubercular therapy (ATT) was started. Patient improved clinically and is being followed up for any neurological deficit. 


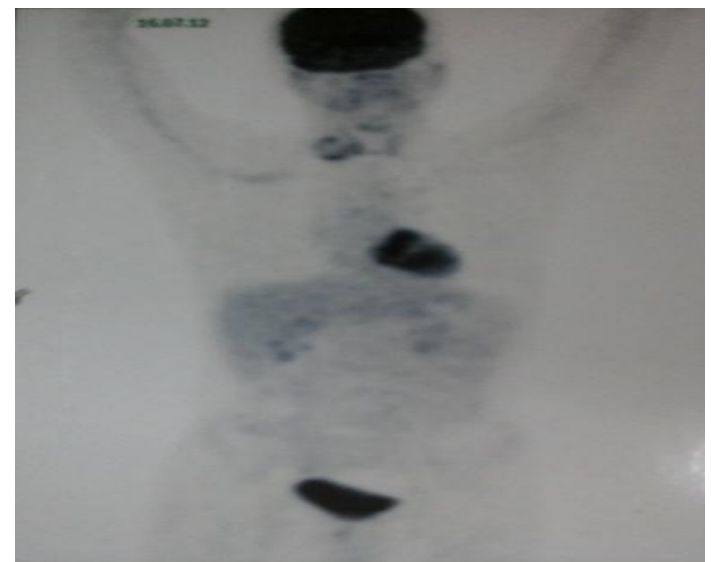

Fig. 1: PET-CT Image Positive for Cervical Lymph Nodes

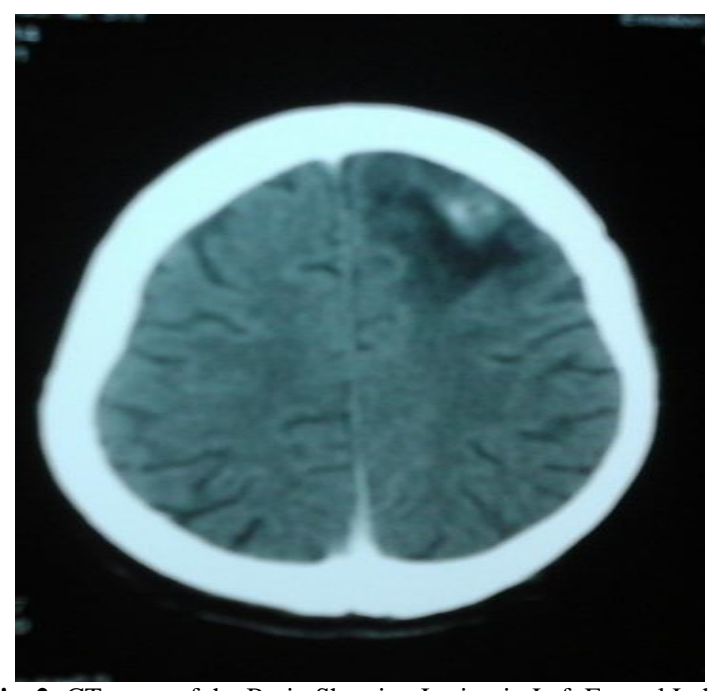

Fig. 2: CT scans of the Brain Showing Lesion in Left Frontal Lobe
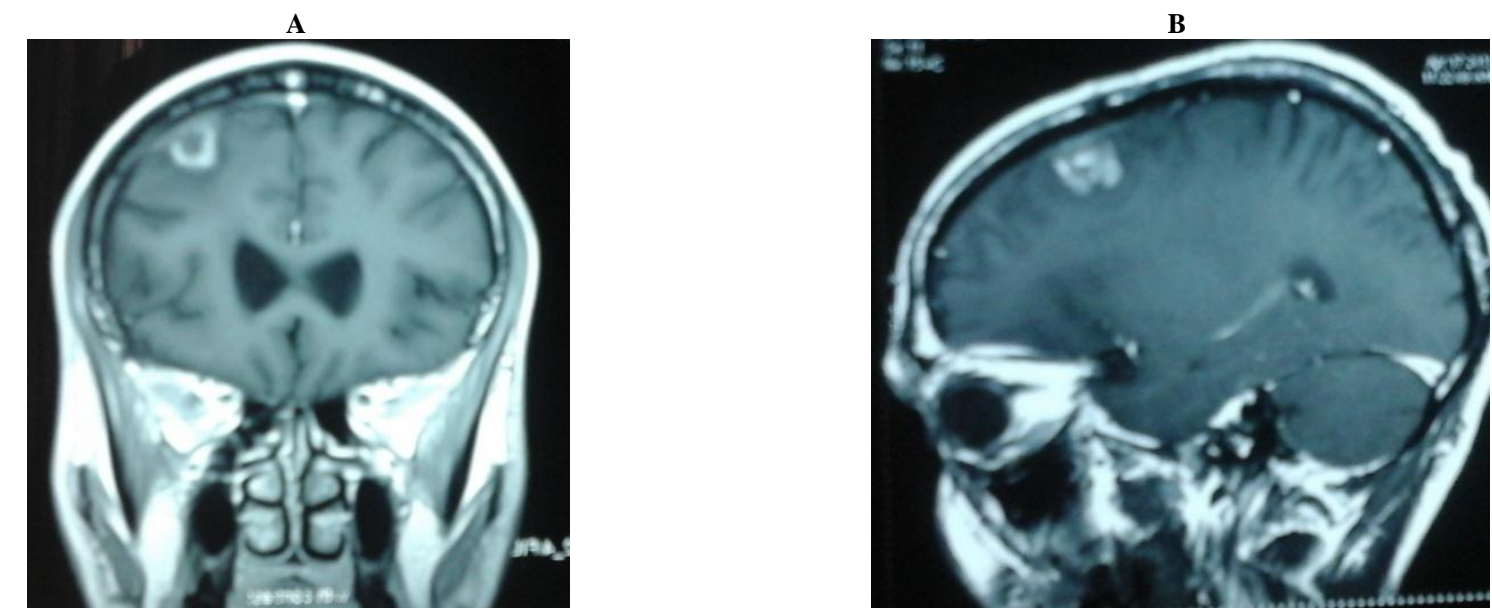

Fig. 3: Contrast Enhanced Mri Brain Images Showing Ring Enhancing Lesion in Left Frontal Lobe, A-Coronal View, B- Sagittal View

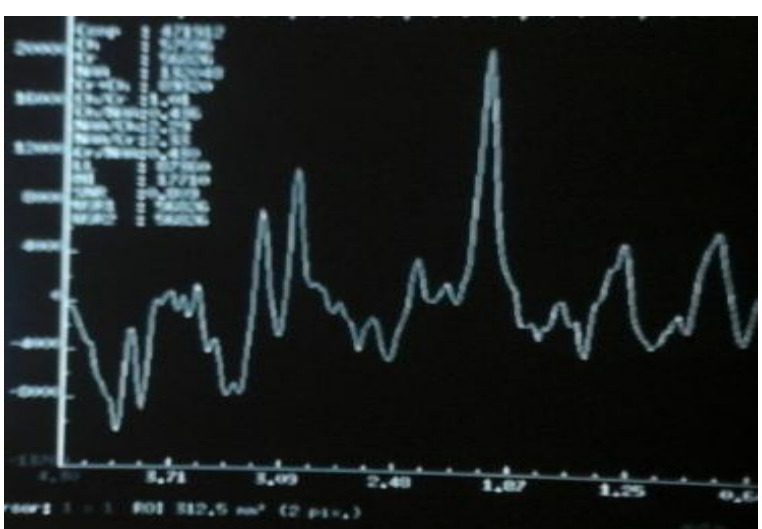

Fig. 4: MR Spectroscopy of the Lesion Showing Lipid Peak Suggestive of Infective Pathology 


\section{Discussion}

The frequency of CNS involvement in TB varies from $0.5 \%$ to 5\%. (Flannery et al, 1993; Bell and Sachs, 1988). Tuberculoma is encountered in 15 to $30 \%$ cases of CNS tuberculosis and is mostly hemispheric (Arvind et al, 1993; Mitchell et al, 1994). The mean age of patients presenting with CNS TB is about 20 years, however, the age may vary from 6 months to 80 years (Gautam, Singh, Khurana, 2013). In this patient the definitive diagnosis of CNS TB was delayed for about 2 years. The mean duration of presentation of CNS TB about 7 months, however, the duration may vary from 15 days to 4 years (Gautam, Khurana, Singh, 2013).

In this case there was no history of pulmonary Koch's or any history of tubercular contact and chest X-Ray was normal. CNS tuberculoma occur secondary to a primary infection elsewhere in the body. Infection spreads hematogenously (Mitchell et al, 1994; Okada et al.1993; Ramamurthi, 1990) and spread occurs primarily from the lungs but can also orginate from TB of the kidneys, tuberculous endometritis, tuberculous ostietis, tuberculous lymphadenitis (Komsuoglu et al, 1994; Vengsarkar et al, 1986 Lassman \& DeAngelis, 2003). A history of tuberculous meningitis is not necessary for the diagnosis of tuberculoma. About half of the patients with intracranial tuberculoma have a history of tuberculosis or current evidence of tuberculosis (Komsuoglu et al, 1994). Twenty five percent of patient show evidence of concomitant tuberculosis lesion elsewhere in the body.

Intracranial tuberculoma is difficult to diagnose when patient has no evidence or history of tuberculous infection and it can occur in otherwise healthy individual. The AFB staining, culture and sensitivity testing and histopathological examination of the granulation tissue may not be possible in such cases and only Chest X-ray, ESR, FNAC from lymph nodes and sputum examination may provide some support to the diagnosis of CNS TB. There should be high index of suspicion for TB in a patient who is from an endemic region. It should always be considered in differential diagnosis of solitary intracranial masses. CT scan or MRI of the brain with MR spectroscopy is a sensitive neuroradiological tool for the evaluation of a patient with intracranial tuberculoma or brain metastasis.

CNS metastasis is a common complication occuring in $10-30 \%$ of cancer patient (Norden,Wen,Kesari, 2005). Among adults most common metastasis include lung cancer $(50 \%)$, breast cancer (15$20 \%$ ), and melanoma (10\%). The next most frequent sources include renal cancer, colorectal cancer, lymphoma and tumors of unknown primary (Kaal and Niel Vech, 2005; Bradleyand Mehta, 2004; Plotkin and Wen, 2003; Patchell, Tibbs and Walsh, 1990). Single metastases account for one third to one quarter of patients with brain metastases (Okada ET al.1993).

Squamous cell carcinoma is the commonest type of cancer of the tongue. CNS Metastases from carcinoma of the tongue is relatively uncommon. Surgery and radiotherapy is the treatment of choice for treating carcinoma of the tongue (Mantsopoulos, et al. 2014 Cheng \& Vincent, 2006).

The imaging findings of intracranial tuberculomas are nonspecific and they have to be differentiated from other causes of space occupying lesions such as high grade gliomas, pyogenic abscess, metastasis, lymphoma, etc. MRI brain with contrast with MRS may be helpful in differentiating tuberculomas from other intracranial mass.

Whole body FDG PET is a sensitive tool for detecting probable primary tumor by visualizing foci of abnormal uptake. In this case PET CT was misleading as the specificity of PET in differentiating malignant tumor from benign or inflammatory lesion is relatively low (Soffetti, et al. 2006).

Biopsy helps to establish the histological diagnosis. The neurosurgical intervention for biopsy may be in the form of stereotactic biopsy or craniotomy and resection of the lesion. Craniotomy and decompression and frozen section biopsy at the time of surgery and histopathological examination of the excised tissue is the treatment of choice in a patient presenting with features of raised intracranial pressure

ATT should be started prior to surgery and cerebral decongestants like acetazolamide, steroids, Glycerol or Mannitol and Frusemide should be advised depending upon the clinical status of the patient and neuroimaging. For follow up CT scan or MRI brain with contrast may be advised.

\section{Conclusion}

CNS tuberculoma is one of the manifestations of extra pulmonary tuberculosis. Its clinical and radiological presentation may be similar to other intracranial space occupying lesions. Newer diagnostic modalities like MRI or PET may not distinguish brain tuberculomas from other lesions of the brain like metastasis. Therefore, a detail clinical and radiological work up followed by neurosurgical intervention is often required to diagnose neurotuberculosis.

\section{References}

[1] Gautam V.K.S, Singh R, Khurana S. Brainstem tuberculoma presenting as stroke. IOSR Journal of Dental \& Medical Sciences: 2279-0853. Vol 4 Iss 6, 2013, pp 18-19.

[2] Gautam V.K.S, Khurana S, Singh R. Diagnostic and therapeutic challenges in the surgical management of CNS tuberculoma, Int J MedHealth Sci. April 2013, Vol-2; Issue-2, pp 161-169.

[3] Ramamurthy B (1970). Intracranial SOL in Souveniour of $20^{\text {th }}$ Anniversary celebration. Institute of Neurology, Madras, 39.

[4] Norden AD, Wen PY, Kesari S. Brain metastasis. Current Opinion in Neurology 2005, 18: 654-661.

[5] Flannery MT, Pattani S, Wallach PM, et al. Case report: hypothalamic tuberculoma associated with secondary panhypopituitarism. Am J Med Sci 1993;306:101-103

[6] Bell WE, Sachs AL, Bacterial meningitis. In Baker AB, Baker LH, eds. Clinical Neurology. Vol2. Philadelphia JB Lippincott, 1988: 58-66.

[7] Arvind C, Korath MP, Raveendranadhan K, et al. A retrospective study of 1247 cases of intracranial tuberculomata diagnosed by computerized tomography. J Assoc Physicians India 1993; 41:559-61.

[8] Mitchell RG, Michael S, Harry LD, Leo W: Cerebral tuberculosis with expansion in to brainstem tuberculoma. J Neurosurg 81: 1994, 927-934,

[9] Okada T, Yoshida T, Asai T, Shintani A: Unusual meningeal tuberculoma: Case report. Neurol Med Chir (Tokyo) 33:710-712, 1993. http://dx.doi.org/10.2176/nmc.33.710.

[10]Ramamurthi B: Tuberculoma and syphillitic gumma, in Youmans JR (ed): Neurological Surgery, Vol 6, Philadelphia, Saunders, 1990 pp 3752-3756

[11] Komsuoglu SS, Ozcan C, Ozmenoglu M, Aydin M, Komsuoglu B: Intracranial tuberculoma: Clinical and computarized tomographic findings. Isral J Med Sci 30; 1994,153-157,

[12] Vengsarkar US, Pisipaty RP, Parekh B, Panchal VG, Shetty MN: Intracranial tuberculoma and the CT scan. J Neurosurg 64: 568-574, 1986.

[13]Lassman AB, DeAngelis LM. Brain metastasis. Neurol Clin 2003; 21: 1-23

[14]Kaal EC, Niel Vech CJ. Therapeutic management of brain metastasis. Lancet Neurology, 2005; 4:289-298.

[15]Bradley KA, Mehta MP, Management of brain metastasis. Semin Oncol 2004; 31:693-701.

[16]Plotkin SR, Wen PY. Brain metastases. In: Samuels MA, Feske SK, editors. Office Practice of Neurology, edition 2, Philadelphia: Churchill livingston; 2003. Pp. 1101-1106.

[17]Patchell RA, Tibbs PA, Walsh JW, et al. A randomized trial of surgery in the treatment of single metastases to the brain. N Engl. J Med 1990; Feb 22:322(8):494-500.

[18] Mantsopoulos K, Psychogios G, Künzel J, Waldfahrer F, Zenk J, Iro H. Primary surgical therapy for locally limited oral tongue cancer. Biomed Res Int. 2014; 2014:738716

[19]Cheng $\mathrm{K}$ Ong and Vincent $\mathrm{F} \mathrm{H}$ Chong. Imaging of tongue carcinoma. Cancer imaging.2006; 6(1): 186-193.

[20] Soffetti R, Cornu P, Delattre J. Y, Grant R, Graus F, Grisold W, Heiman J, Hildebrand J, Hoskin P, Kalljo M, Krauseneck P, Marosi C, Siegal T, Vecht C. EFNS Guidelines on Diagnosis and Treatment of brain metastasis: report of EFNS Task Force. Euro J Neurol 2006, 13: 674-681. 